weight and an immunological response indistinguishable from that of the functional species. Proteolysis and reduction lead, as usual, to A and B fragments, indistinguishable in detergent gel electrophoresis from those of the wild type cells, but neither in the A-fragment nor in the nicked unreduced molecule is there any detectable blocking activity towards translocase. Labelled NAD does not bind to the mutant fragment, nor will the latter inhibit complex formation between normal A-fragment, NAD and the translocase. It thus seems likely that the mutation is in the NAD-binding site.

The other end of the toxin molecule - the B-fragment-on the other hand, seems to be fully operative, for it will compete with active toxin on equal terms for the attachment sites on HeLa cells, as shown by the way in which it will prevent it from inhibiting the incorporation of labelled amino-acids into protein. By contrast, the low molecular weight mutant, missing the greater part of the B-chain, is incapable of any such competition.

The clinching experiment now follows: mixtures of the two mutant proteins, one defective in the $A$, the other in the B part, both almost wholly in the form of intact, un-nicked chains, generated a low level of activity on standing, and this was elevated to appreciable levels by the presence of a thiol reagent. Uchida et al. inferred that fragment hybridization by disulphide exchange was going on within the nicked fraction of the protein, and indeed when the protein was treated with trypsin and then the thiol, the level of toxicity which was generated increased greatly. In the absence of thiol, electrophoresis shows only two important components, corresponding to the total chains of 62,000 and 45,000 molecular weight, enzymatic activity being associated with the latter. After exposure to the thiol, and reoxidation, enzymatic activity appeared in the higher molecular weight zone to an extent corresponding to about half the concentration of this species. The toxicity in animals after this treatment was $40-80$ per cent of the calculated maximal value, and could be specifically inhibited by antitoxin. It is thus established that the part of the chain corresponding to the B-fragment is indeed implicated in penetration of the cell membrane, and opens the door, so to speak, for entry of the instrument of destruction, the A-fragment.

From the nature of the B-mutation, Uchida et al. had deduced that this fragment occupies the C-terminal part of the intact toxin chain. Michel et al. (Biochim. Biophys. Acta, 257, 249 ; 1972) have now confirmed this directly. They have separated the fragments from the nicked toxin, and determined the end groups of both. Assuming, as it is reasonable to do, that the naturally generated fragments can be identified with the products of trypsin treatment, it turns out that the A-chain has a glycine $\mathrm{N}$-terminus, as for the most part has the intact toxin, whereas from the B-chain four N-terminal species can be isolated, among which serine and glycine predominate. At the C-terminal end, the toxin has arginine, and the A-chain arginine and histidine in a ratio of $2: 1$, whereas the B-chain has a variety of amino-acids. The sequence of the first fifteen $\mathrm{N}$-terminal residues of the $\mathrm{A}$ chain is also reported.

The endogenous protease thus evidently produces untidy ends, and the A-chain in fact shows electrophoretic microheterogeneity. The formation of new C-termini in the A-chain and $\mathrm{N}$ termini in the B-chain establishes the C-terminal position of the latter in the toxin. The prevalence of $\mathrm{C}$-terminal arginines suggests a trypsin-like specificity of the protease. It must be supposed that $\mathrm{A}$ and $\mathrm{B}$ are globular entities joined by an exposed segment of chain, which is vulnerable to proteolysis. Some amino-acids must be presumed lost in the digestion process, but the additivity of the molecular weights of the $A$ and $B$ pieces indicates that these cannot comprise more than a few residues. There must be at least two arginines in this connecting segment, one flanked by a glycine on the carboxyl side, the other by a serine. The presence of small amounts of three $\mathrm{N}$-terminal amino-acids besides glycine in the intact toxin is unexplained.

\section{PALAEOBOTANY \\ Wealden Fern}

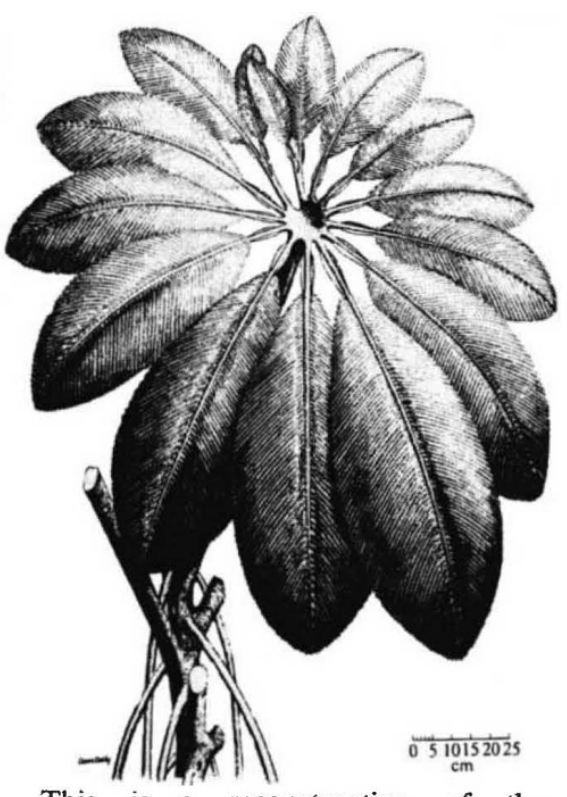

This is a reconstruction of the Cretaceous fern Weichselia reticulata which has been prepared by Mrs C. Dalby for K. L. Alvin's new monograph on specimens of the species collected from the Wealden of Belgium (Mem. Inst. Roy. Sci. Nat. Belg., No. $166 ; 1971)$. The figure, about $1 / 25$ natural size, shows the stem with petioles, the rooting organs (unique among ferns), the aerophores and one complete vegetative frond.

\title{
Cyclic AMP and Cell Adhesion
}

Transformed cells growing in tissue culture exhibit a wide range of phenotypic traits not shown by cultures of comparable but untransformed cells. Transformants, for example, have a changed morphology and a changed cell surface; they usually continue to multiply in serum concentrations too low to support the multiplication of untransformed cells; they adhere less tenaciously to their substratum than untransformed cells and sometimes grow in suspension culture; and, most importantly, they grow to very much higher saturation densities than their untransformed counterparts. In other words transformed cells are less susceptible to contact inhibition of growth.

Over the past several months it has been shown that when appropriate concentrations of cyclic AMP and of theophylline, which inhibits cyclic AMPase, are added to cultures of transformed cells many of these phenotypic traits are reversed and the transformants begin to behave as if they had not been transformed. In Nature New Biology next week (April 26) Johnson and Pastan add the tenacity of adhesion of fibroblasts to their substratum to the growing tally of characters which can be changed by manipulating the concentration of cyclic AMP and theophylline.

Johnson and Pastan have taken cultures of mouse fibroblasts, L-929 cells and SV40 transformed 3T3 cells and measured the time required to detach 50 per cent of the cells in a culture with 0.02 per cent trypsin in the presence or absence of cyclic AMP and theophylline. Without the additives half the L-929 cells and SV3T3 cells growing in Johnson and Pastan's conditions are detached in $16 \mathrm{~min}$ and 12 min respectively, while after $30 \mathrm{~min}$ or 3 days' incubation in the presence of cyclic AMP and theophylline these times are increased to $47 \mathrm{~min}$ and 22 min respectively. Furthermore, this increased adhesiveness of mouse fibroblasts growing in the presence of cyclic AMP is not inhibited by cycloheximide but it is antagonized by serum and is fully reversible. 